\title{
ASO Author Reflections: Prognostic Index after Neoadjuvant Chemotherapy (PINC) in Pancreatic Ductal Adenocarcinoma as Novel Tumor Regression Grading System
}

\author{
Miriam Redegalli, $\mathbf{P h D}^{1,2}$ (1), and Marco Schiavo Lena, $\mathbf{M D}^{1,2}$ \\ ${ }^{1}$ Pathology Unit, IRCCS San Raffaele Scientific Institute, Milan, Italy; ${ }^{2}$ Vita-Salute San Raffaele University, Milan, Italy
}

\section{PAST}

Pancreatic ductal adenocarcinoma (PDAC) is one of the most lethal malignancies. Although surgery is the mainstay of therapy, up to $80 \%$ of patients relapse after curative resection, suggesting the presence of systemic diffusion in the early phases of the disease. ${ }^{1}$ Several clinical trials have evaluated a preoperative therapeutic strategy, demonstrating that neoadjuvant chemotherapy (NACT) has a positive impact on patient outcomes compared with upfront surgery followed by adjuvant chemotherapy. NACT is known to induce a spectrum of histological changes in PDAC. Many tumor regression grading (TRG) systems have been developed, however they lack precision and accuracy, questioning their clinical utility. ${ }^{2}$ In this study, we sought to identify objective morphological parameters that portend prognostic value in PDAC patients resected after NACT to develop a prognostic index.

\section{PRESENT}

In the present study, we evaluated 20 morphological features in 105 patients diagnosed with resectable or borderline resectable PDAC, of whom 69 were treated with NACT and 36 with upfront surgery followed by adjuvant chemotherapy. ${ }^{3}$ The distribution of the parameters analyzed was significantly different between the upfront surgery and the NACT group. On multivariate analysis for

(C) Society of Surgical Oncology 2022

First Received: 2 February 2022

Accepted: 2 February 2022;

Published Online: 20 February 2022

M. Redegalli, $\mathrm{PhD}$

e-mail: redegalli.miriam@hsr.it patients who received NACT, perineural invasion and lymph node ratio predicted shorter overall survival (OS) and disease-free survival (DFS), while a high stroma-toneoplasia ratio predicted longer OS and DFS. The three parameters were combined to generate a prognostic index (PINC) that significantly distinguished patients with poor outcome from patients with a better prognosis.

\section{FUTURE}

These findings suggest that the PINC is a reliable TRG system to predict patient outcomes after NACT followed by surgery for PDAC. To verify the robustness of the PINC, a validation study on a larger cohort of patients will be carried out.

DISCLOSURES Miriam Redegalli and Marco Schiavo Lena report no conflicts of interest.

\section{REFERENCES}

1. Kim YI, Song KB, Lee YJ, et al. Management of isolated recurrence after surgery for pancreatic adenocarcinoma. Br J Surg. 2019;106(7):898-909. https://doi.org/10.1002/bjs.11144.

2. Janssen BV, Tutucu F, van Roessel S, et al. Amsterdam International Consensus Meeting: tumor response scoring in the pathology assessment of resected pancreatic cancer after neoadjuvant therapy. Mod Pathol. 2021;34(1):4-12. https://doi.org/10. 1038/s41379-020-00683-9.

3. Redegalli M, Schiavo Lena M, Cangi MG, Smart CE, Mori M, Fiorino C, et al. Proposal for a new pathologic prognostic index after neoadjuvant chemotherapy in pancreatic ductal adenocarcinoma (PINC). Ann Surg Oncol. 2022. https://doi.org/10.1245/ s10434-022-11461-z.

Publisher's Note Springer Nature remains neutral with regard to jurisdictional claims in published maps and institutional affiliations. 